\title{
Asymmetric skeletal anomalies in siblings
}

\author{
Wayne S. Stanley ${ }^{1 *}$, Mason Barr, $J_{R}{ }^{2}$, Robert Hensinger ${ }^{3}$, Stephen G. Ruby ${ }^{4}$, Daniel L. Van \\ DYKE ${ }^{1}$ AND LESTER WEISS ${ }^{1}$ \\ ${ }^{1}$ Medical Genetics and Birth Defects Center, Henry Ford Hospital, Detroit, Michigan, Departments \\ of ${ }^{2}$ Pediatrics and Communicable Diseases, University of Michigan Medical School, Ann Arbor, \\ Michigan, ${ }^{3}$ Orthopaedic Surgery, University of Michigan Medical School, ${ }^{4}$ Pathology, Saint John \\ Hospital, Detroit, Michigan; *Current address: Section of Cytogenetics, Department of Pathology, \\ Medical University of South Carolina, Charleston, SC U.S.A.
}

\begin{abstract}
We describe two siblings with asymmetric limb reduction malformations. Such anomalies are usually considered to result from sporadic events, but the recurrence in siblings without any identifiable teratogenic insult suggests a genetic etiology. This finding becomes important when parents are counseled about future pregnancies. The use of prenatal diagnostic techniques during subsequent pregnancies should be considered.
\end{abstract}

Received 16 May 1983, revised, accepted for publication 19 January 1984

Key words: Asymmetric skeletal malformation; genetic counseling; Mendelian inheritance.

Limb abnormalities can result from monoor polygenic effects (Temtamy \& McKusick 1978), numerical and structural chromosome abnormalities (Bofinger et al. 1973), teratogenic influences (Speirs 1962), or vascular disruptions (Johnson \& Robinow 1978, Marin-Padilla et al. 1981). Although bilateral limb malformations are often ascribed to genetic or teratogenic factors, unilateral birth defects usually have a nongenetic origin. As a result, parents of a child with unilateral limb defects are usually counseled that the risk of recurrence is low.

We report two siblings, born to nonconsanguineous parents, who had asymmetric malformations of the upper and lower extremities. When we were unable to identify nongenetic causes for recurrence, we were led to suspect genetic factors.

\section{Case Reports}

Case 1

The female proband was delivered by Cesarean section because of a breech presentation in a primigravida mother. She weighed $2510 \mathrm{gm}$ at birth (Apgars; 9 and 9 at 1 and $5 \mathrm{~min}$ ). Multiple anomalies at birth consisted of underdeveloped and malformed extremities on the right side. Neither respiratory distress nor feeding problems occurred during the immediate postpartum period.

Both parents were 27 years old. The mother denied drinking alcohol, smoking, or $\mathrm{x}$-ray exposure at any time during pregnancy. She had brief episodes of spotting during the sixth gestational week and between the second and third month of gesta- 
tion. She took doxylamine succinate (Bendectin) for nausea. The parents are unrelated, and neither parent has a family history of limb malformations or other birth defects.

At 11.5 months the child's head circumference was $46 \mathrm{~cm}$; she was $73 \mathrm{~cm}$ long and weighed $6350 \mathrm{gm}$. Her face was not dysmorphic, but her head was slightly asymmetric with left parietal prominence. Examination of the ears, eyes, nose and oral cavity was unremarkable. The neck had full range of motion. Lung and cardiovascular examinations were normal. The abdominal wall muslces were lax and the umbilicus was displaced $2-3 \mathrm{~cm}$ to the right of the midline. The right nipple was smaller than the left, and the mass of the right pectoralis muscle was smaller than the left. The right clavicle was also much smaller than the left. The muscle mass of the right upper extremity was less than on the left, and the right hand was appreciably smaller than the left. The function of the right arm and hand, however, appeared to be intact.

The right lower extremity was small and bowed. The range of motion of the right hip joint was diminished in flexion, extension, and rotation. The patient was unable to bear weight on her right lower extremity and the muscle tone was diminished. Her deep tendon reflexes were symmetrical and normally active. The patient's developmental assessment demonstrated no intellectual impairment.

Roentgenographic examination demonstrated that the right clavicle, humerus, ulna, radius, carpal and digital bones were small compared to those on the contralateral side. The entire right hemipelvis was
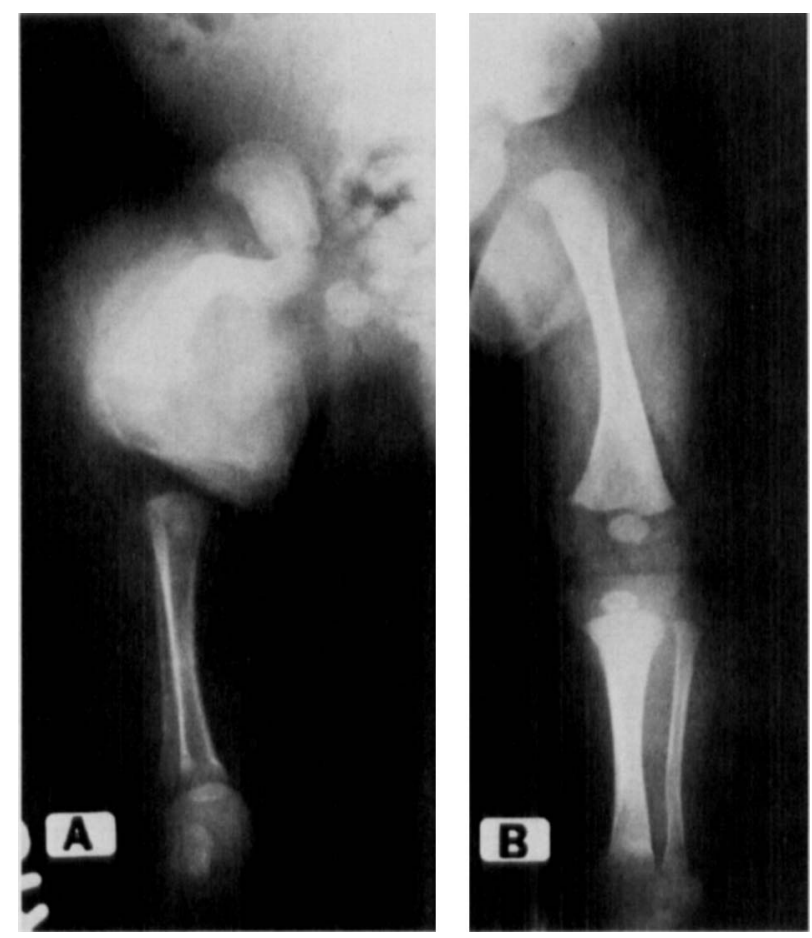

Fig. 1. (A) Bowed and shortened right femoral bone of proband. (B) Left leg of proband. 
smaller, with no recognizable acetabulum. The right ischium was absent, and the pubis was small. The right iliac wing was flaired. The sacrum and scaroiliac joints appeared normal. The right femoral shaft was bowed, shortened, and did not appear to articulate with the pelvis. Proximally, the femoral shaft remnant was dislocated into the region of the inguinal ligament and abdomen. The right tibia was smaller than the left and had a mild anterior bow (Fig. 1). The ossification of the right os calcis and talus was retarded compared to the left, but otherwise they appeared normal. An intravenous pyelogram and voiding cystourethrogram were normal. Ultrasonography revealed an eventration of the right hemidiaphragm. Peripheral blood GTG-banded midmetaphase and prometaphase karyotypes were normal.

When the parents sought genetic counseling at two medical centers, they were advised that asymmetric malformations of this type usually result from an intra-uterine insult that occurs before the eighth week of gestation. They were also counseled that recurrences have not been reported and that the risk to future pregnancies is low. To exclude the possibility of a uterine abnormality as the cause, the mother had an ultrasound examination of her uterus and a hysterosalpingogram. Both studies showed normal uterine anatomy.

\section{Case 2}

The second pregnancy was uncomplicated except for nausea, and a mild gastrointestinal illness between the second and third month of gestation. The mother took no medications except vitamins. No teratogenic factors were identified in the occupational or home environment.

A $1080 \mathrm{gm}$ male infant was delivered by Cesarean section at 31 weeks of gestation following spontaneous onset of labor. The infant had multiple congenital abnormalities and no spontaneous respirations or

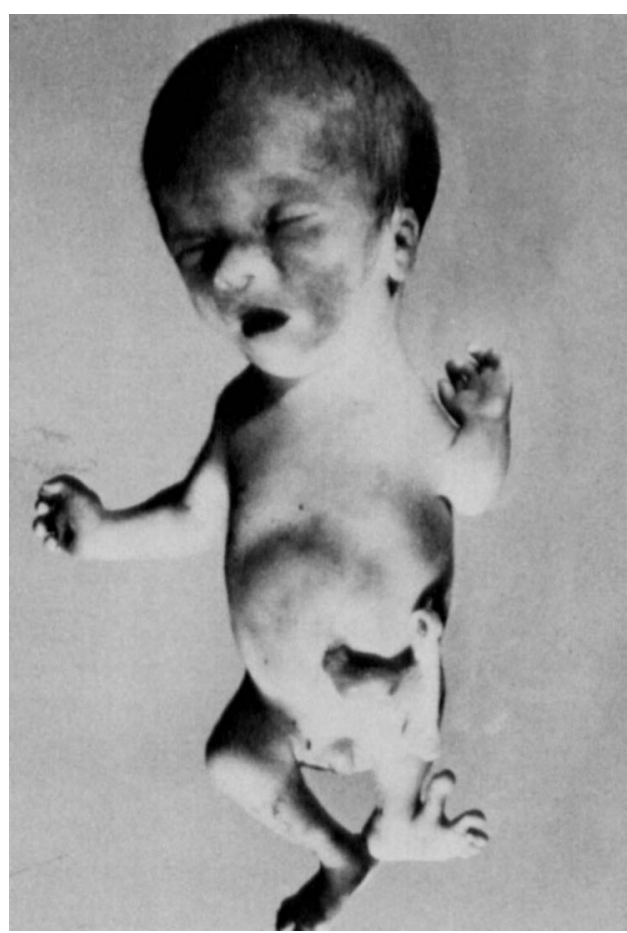

Fig. 2. Case 2. Limb abnormalities are evident on the left side.

heartbeat. The baby remained unresponsive and was pronounced dead approximately 40 minutes after delivery.

The external abnormalities included cleft palate, low set ears, a broad webbed neck, small left extremities, bilateral simian creases, and rocker bottom feet (Fig. 2). Visceral abnormalities included membranous right hemidiaphragm and absence of the left hemidiaphragm with hypoplasia of the left lung and herniation of the liver, spleen, pancreas and gastrointestinal tract into the left pleural cavity. There was a horseshoe kidney with herniation into the right pleural cavity, anterior placement of the anal orifice, hypospadias and cryptorchidism.

Roentgenographic examination showed that the humerus on the left was shorter 
than that on the right, and the shoulder girdle was smaller than normal. The humerus did not appear to articulate normally with the scapula, which was small. The glenoid fossa and acromial process were absent. The left radius was approximately half the length of the right. The ulna was bowed and shortened. The bones of the hand, though not well ossified, appeared normal (Fig. 3). The entire left hemipelvis, femur, and fibula were absent as was the right side of the sacrum. Part of the right iliac bone, the acetabulum and the fibula were absent. The bones of the feet were not well ossified.

On lateral view of the skull and spine, the vertebrae appeared normal. Similarly, the skull, mandible, and sternum, although poorly ossified, appeared to be normal. GTG-banded midmetaphase karyotypes prepared from blood and soft tissue obtained at autopsy were normal.

\section{Discussion}

The asymmetric skeletal anomalies in these two siblings are unusual and may represent a new malformation syndrome.

Asymmetric limb defects do occur in the oro-mandibular-limb hypogenesis syn- dromes, but these always include abnormalities of the face, jaws, mouth, and tongue. The etiology of these syndromes is unclear but may be secondary to vascular disruption (Johnson \& Robinow 1978). The Poland sequence exhibits unilateral brachysyndactyly of the upper extremity and absence of the ipsilateral pectoral muscle. The etiology of this syndrome is not known and is usually sporadic (Ireland et al. 1976). Unilateral asymmetry is also a feature of Silver syndrome, which includes a small triangular face, downturned corners of the mouth and large fontanels (Silver 1964). Our two cases differ from these syndromes.

Marin-Padilla et al. (1981) reported a 1518-week-old female fetus with unilateral arm and leg abnormalities that may have resulted from vascular disruptions. While it is possible that the limb abnormalities in our two cases might have a vasculopathic etiology, the recurrence in siblings reduces this likelihood.

Pseudothalidomide (Roberts) syndrome is an autosomal recessive condition characterized by deformities of upper and lower limbs associated with bilateral cleft lip and severe mental retardation (Freeman et al. 1974). These latter features were not observed in our cases. Hecht \& Scott (1981)

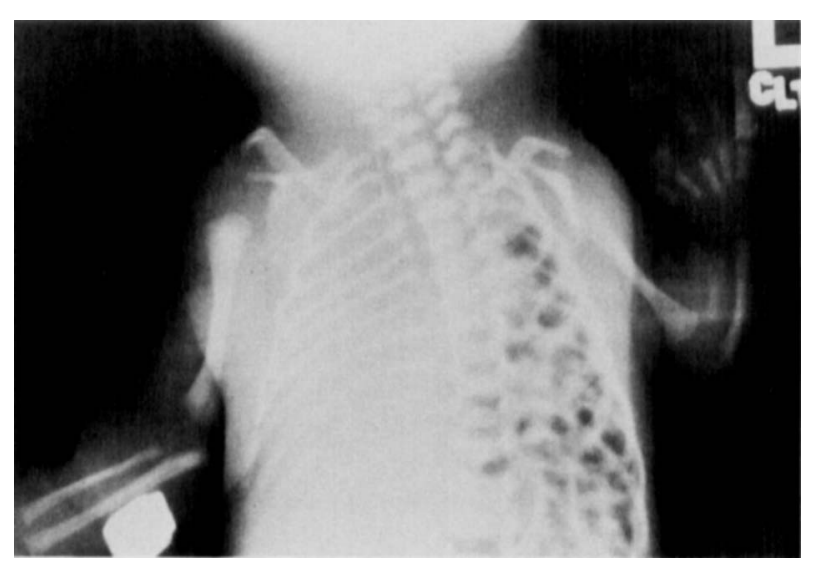

Fig. 3. Case 2. Herniation of the abdominal contents into the left pleural cavity. Left humerus and shoulder girdle are smaller than normal, left ulna and radius are shortened. 
described two brothers born with unilateral hand malformations, which consisted of shortened digits due to complete or partial absence of the hand bones. The authors indicate that the recurrence of these specific anomalies in siblings of consanguineous parents suggests autosomal recessive inheritance.

Falek et al. (1968) reported a unique unilateral pattern of malformations in two female siblings who exhibited moderate to severe hypomelia, cardiovascular abnormalities, and scaling of the skin on the left side with a sharp midline demarcation. Our cases have similar limb reduction malformations but lack the skin hypoplasia and cardiac defects associated with that syndrome.

Autosomal recessive inheritance is suggested for our cases because there was a negative family history of skeletal malformations, an absence of any identifiable teratogenic exposure, and the occurrence of opposite sex in the siblings.

In conclusion, in isolated cases of asymmetric skeletal malformations where an environmental insult cannot be identified and a vascular accident is unlikely, a genetic etiology should be considered. The parents should be counseled about this possibility, and prenatal imaging techniques may be warranted during subsequent pregnancies.

\section{Acknowledgments}

We wish to thank Dr. Patricia L. Cornett for editorial assistance and Dr. V. Ramesh Babu for preparation of prometaphase chromosomes.

\section{References}

Bofinger, M. K., P. S. J. Dignan, R. E. Schmidt \& J. Warkany (1973). Reduction malformations and chromosome anomalies. Am. J. Dis. Child. 125, 135-143.

Falek, A., C. W. Heath, A. J. Ebbin \& W. R. McLean (1968). Unilateral limb and skin deformities with congenital heart disease in two siblings: A lethal syndrome. J. Pediatr. 73, 910913.

Freeman, M. V. R., D. W. Williams, N. Schimke, S. A. Temtamy, E. Vachier \& J. German (1974). The Roberts syndrome. Clin. Genet. 5, $1-16$.

Hecht, J. T. \& C. I. Scott, Jr. (1981). Recurrent unilateral hand malformations in siblings. Clin. Genet. 20, 225-228.

Ireland, D. C. R., N. Takayaona \& A. E. Flatt (1976). Poland's syndrome: A review of 43 cases. J. Bone Joint Surg. 58A, 52-58.

Johnson, G. F. \& M. Robinow (1978). AglossiaAdactylia. Radiology 128, 127-132.

Marin-Padilla, M., J. M. Graham, Jr. \& G. M. Simmons, Jr. (1981). Extrinsic vascular disruptions and unilateral limb malformations. Conference on Malformations and Morphogenesis, Hanover, New Hampshire, USA.

Silver, H. K. (1964). Asymmetry, short stature and variations in sexual development. A syndrome of congenital malformations. Am. J. Dis. Child. 107, 495-515.

Speirs, A. L. (1962). Thalidomide and congenital abnormalities. Lancet i, 303-305.

Temtamy, S. A. \& V. A. McKusick (1978). The Genetics of Hand Malformations. New York, Alan R. Liss, Inc.

\section{Address:}

Dr. Wayne Stanley Section of Cytogenetics Department of Pathology

Medical University of South Carolina

171 Ashley Avenue

Charleston, SC 29425

USA 